\title{
Airborne hyperspectral discrimination of tree species with different ages using discrete wavelet transform.
}

\begin{abstract}
In this article, the capability of discrete wavelet transform (DWT) to discriminate tree species with different ages using airborne hyperspectral remote sensing is investigated. The performance of DWT is compared against commonly used traditional methods, i.e. original reflectance and first and second derivatives. The hyperspectral data are obtained from Thetford forest of the UK, which contains Corsican and Scots pines with different ages and broadleaved tree species. The discrimination is performed by employing three different spectral measurement techniques (SMTs) including Spectral Angle Mapper (SAM), Spectral Information Divergence (SID), and a combination of SAM and SID. Five different mother wavelets with a total of 50 different orders are tested. The wavelet detail coefficient (CD) from each decomposition level and combination of all CDs plus the approximation coefficient from the final decomposition level (C-All) are extracted from each mother wavelet. The results show the superiority of DWT against the reflectance and derivatives for all the three SMTs. In DWT, C-All provided the highest discrimination accuracy compared to other coefficients. An over- all accuracy difference of about $20-30 \%$ is observed between the finest coefficient and C-All. Amongst the SMTs, SID provided the highest accuracy, while SAM showed the lowest accuracy. Using DWT in combination with SID, an overall accuracy up to around $71.4 \%$ is obtained, which is around $13.5 \%, 14.7 \%$, and $27 \%$ higher than the accuracies achieved with reflectance and first and second derivatives, respectively.
\end{abstract}

